\title{
The Research of University Scientific Research Evaluation Construction System Based on Qualitative and Quantitative Analysis Method*
}

\author{
Hailing Jiang \\ College of Tourism and Geographical Science \\ Jilin Normal University \\ Siping, China 136000
}

\author{
Xiuhua Zhang \\ College of Tourism and Geographical Science \\ Jilin Normal University \\ Siping, China 136000 \\ Shixin Zheng \\ College of Tourism and Geographical Science \\ Jilin Normal University \\ Siping, China 136000
}

\section{Shuang Li}

College of Tourism and Geographical Science Jilin Normal University

Siping, China 136000

\author{
Xinyu Wang \\ College of Tourism and Geographical Science \\ Jilin Normal University \\ Siping, China 136000 \\ Yue Li \\ College of Tourism and Geographical Science \\ Jilin Normal University \\ Siping, China 136000 \\ Bowen $\mathrm{Fu}$ \\ College of Tourism and Geographical Science \\ Jilin Normal University \\ Siping, China 136000
}

\begin{abstract}
Based on the data of scientific research evaluation indexes of universities and the methods of quantitative and qualitative analysis. A quantitative analysis model is expected to be established in scientific research evaluation system, which can clarify some issues, for example, the impact of scientific research evaluation indexes on the ranking of colleges. In following work, from the relationship between university evaluation and scientific evaluation, university scientific research classification evaluation and university scientific research evaluation, the basic situation of university research evaluation needs to be clarified in China, which can lay a theoretical foundation for the in-depth study of the university scientific research evaluation system.
\end{abstract}

Keywords-the construction of scientific research evaluation system in universities; quantitative analysis model method; qualitative analysis model method; university ranking

\section{INTRODUCTION}

For scientific research of research university, effective research management play an important role in improving

*Fund sponsored project: The education department project of Jilin province (Jijiaokewenhezi No.172 [2016]), the national natural science foundation of China (41701424), the open research fund project of national key laboratory of remote sensing science (OFSLRSS201716). scientific research level and enhancing scientific research strength. Meanwhile, it play an important role in the quality of scientific research activities, the efficiency of scientific research activities of research universities is scientific research management, and the development of science and technology in China. Besides, Scientific and technological innovations contribute to improve social productive force and enhance comprehensive national power [1]. The implementation of innovation-driven development strategy can promote social progress and sustainable economic development. As the main base for talents cultivation and science and technology innovations, colleges and universities are the source and main part for promoting technological innovation, technological progress and economic development. In recent years, the scientific research of universities is regard as key point by the Ministry of Education and the Provincial Department of Education, which means to accelerate Constructing and Perfecting the Research Innovation System of Colleges and Universities and Deepening the Reform of Scientific Research Evaluation in Colleges and Universities [1] [2] [3]. As an important part of the scientific research work of colleges and universities, the scientific research evaluation system of colleges is consisted of three parts: scientific research results, scientific research bases and scientific research projects, which layer a 
foundation for the comprehensive level of university development. According to the analysis results of evaluation index system and weights in the 2014 China University Rankings, that released by the China Alumni Association, in the ranking, the outstanding alumni and scientific research achievements are used as the most important indexes in cultivation of talents, showing that the scientific research achievements play an key role in accelerating the development of colleges [2] [3].

In the past, scientific research evaluation system was constructed by summing up experience, instead of considering the relationship between science and education development. In recent years, the construction of scientific research evaluation system has a rapid development, becoming an active research field in management science and other research fields [1] [4]. Some academic papers have been published. For example, "Research on Research Management Evaluation System of Research Universities". Its research results provide scientific and advanced methods for scientific analysis, technology decisions, economics and societies, and close the relationship between the construction research evaluation system construction and the education development of the times.

The original evaluation system was improved by combining quantitative and qualitative analysis. Firstly, There are five steps: a list of indexes, the selection of experts, advisory, statistical analysis, the index system; Secondly, the principles includes systematicity and comprehensiveness, classification and evaluation, scientificity and reasonability, oriented and dynamic quality, independence and concise, operability and comparability; Thirdly, the indexes is selected in actual situations of the evaluation points that reflect evaluated objects objectively; Fourthly, The weights are calculated by indexes value that show the importance of each index in the index evaluation system; Finally, a complete research evaluation system was constructed [5].

The real purpose of carrying out research evaluation work is to summarize achievements and experiences through evaluation, instead of rankings. According to evaluations of various universities, Problems in scientific research can be found. Then, the key problems in the university education system can be improved in a targeted manner. Thus, research and comprehensive strength of universities can be improved.

\section{The Quantitative Analysis Model MethoD}

\section{A. Correlation Analysis Model Method}

Correlation analysis is a statistical analysis method that studies the correlation between two or more indexes with equal status. In the process of constructing the research evaluation system, the correlations between the indexes lower the scientificity and validity of the evaluation index system. For example, there is a certain probability of reassignment on relevant indexes and the reuse of evaluation objects. According to relevant analysis model method, Judging and evaluating the dependencies between indexes, the related direction and extent of the indexes can be determined. Then deleting some indexes with higher correlation coefficient values, the evaluation objects information can be avoided from reusing at a certain level [4] [5] [6].

\section{B. Discriminative Power Analysis Model Method}

The discriminative power analysis is the ability of the evaluation index to distinguish the characteristic difference of the evaluated object, which is an objective existence problem that needs to be solved by the scientific evaluation system. According to university research evaluation index, the discriminative power analysis model is used in this study, which also called the coefficient of variation model, and the strengths of scientific research can be distinguished for universities with similar scientific research ability in university rankings [7]. The specific performances as follows:

- All the evaluated universities have almost the same score on a certain index, which indicates that the evaluation index has very low discriminative power, and has no meaning to evaluate the difference of scientific research ability among different universities

- All evaluated universities show an obvious difference scores on a certain index, which proves that the evaluation index has a high discriminating ability for evaluating the difference in scientific research ability of different universities.

\section{The Analytic Hierarchy Process}

The Analytic Hierarchy Process (AHP) is used to determine the weight coefficients of each evaluation dimension and evaluation index in the university research evaluation system. Firstly, a hierarchical structure (secondary index) needs to be established that describes the internal independence of the scientific evaluation system. The relative importance between two indexes is compared (calculate weights), which gives the corresponding scale, and the Judgment matrix of the upper index to the lower index is constructed [8]. Secondly, judging the third-level indexes under the second-level indexes, the relatively importance sequences are obtained that is the three-level indexes in their corresponding second-level indexes. The analytic hierarchy process can construct the evaluation index system more easily and reasonably, and have obvious effects on some complex problems that are difficult to completely deal with quantitative methods. Its process can be concluded as follows: decomposition, judgment, synthesis. Based on the excellent characteristics of systematic, concise practical and applicability, The Analytic Hierarchy Process, reflecting the basic characteristics of people's good decision-making thinking, wins the world's wide-ranging adoption. [6] [8]

\section{Principal Component Analysis Model Method}

In the case that the comprehensive impact analysis has more evaluation indexes, two problems are necessary to be solved: the relevance and weights assignment of the evaluation indexes. The Principal component analysis method is also used to create scientific research evaluation system [9]. The process as follows: firstly, calculating the eigenvalues and eigenvalues of the covariance matrix or the 
correlation coefficient matrix, $\mathrm{m}$ indexes are extracted from the $\mathrm{n}$ original indexes as irrelevant principal components according to the specified contribution rate; Secondly, show the inner relationship between them. This method can not only solve problems of the multi-evaluation indexes correlation and empowerment, but also complete the simplification of the implementation evaluation indexes.

\section{E. Data Envelopment Analysis}

Data envelopment analysis (DEA), a new method, is often used in recent years. Appling the data envelopment analysis method to the dynamic evaluation of scientific research, a DEM model should be constructed firstly according to the comprehensive indexes of scientific research achievements over the years [10]. Constructing the front surface of the data envelopment, the effectiveness of each evaluation unit can be calculated, and the management efficiency of each evaluation unit in different periods can be compared. Through the use of data envelopment analysis for scientific research evaluation, the total improvements of the scientific research quantities can be compared for different universities in differ periods. Meanwhile, the specific status of scientific research can be reflected for different universities in differ periods. Mainly based on analyzing and calculating the statistical data, First of all, this method avoids the interference of human factors. In addition, the impact of the initial scientific research volume of the university can be minimized. Moreover, the comparison of scientific research quantities in different time periods can be reflected [8] [9] [10].

\section{The Qualitative Analysis Model Method}

\section{A. Collective Opinion Method}

In collective opinion method, through research's convening to the relevant person of the enterprise, the individual's contacting of things, marketable cognition, and experiential cognition and so on, the market prospect is judged and predicted, and the results are comprehensively analyzed. In the construction of research evaluation system in universities, the collective opinion method is represented by peer review. First of all, according to different subjects, the evaluated objects are divided into 68 units to be evaluated; then, evaluation results for each unit are obtained by the evaluation of an evaluation group consisting of multiple experts [6]. In the collective opinion method, experts participate in the decision-making of scientific evaluation, which makes up for the lack of cognitive and knowledge base of laymen. Collective opinion method plays a certain important role in scientific research evaluation.

\section{B. Literature Research Method}

In literature research method, through the collection, identification, collation and research of the literature, the facts are recognized scientifically. The literature method is an ancient and vital research method, originally used as a measurement method in the fields of library science and information science. Based on books, periodicals and other formally tangible documents, this method can be used to quantitatively analyze books and other documents, accordingly enhancing the exchange of literature and intelligence [4] [9]. The literature method was widely recognized and applied now, which is also used to statistic and analyze a large number of documents and patents to conduct, and to complete the prediction and evaluation scientific research evaluation in developed countries in1970s. There are Advantages as follows: Firstly, it can be used to study scientific research indexes that are impossible or difficult to access; secondly, literatures have a high authenticity; thirdly, the research process is simple and inexpensive. Fourthly, the research results are highly reliable. In terms of disadvantages: Firstly, literatures are incomplete in themselves, such as document deviation, incomplete records, document damage, etc. Secondly, literatures are different to collect, collate and code.

\section{Expert Meeting Method}

In the Expert Meeting, by inviting relevant experts and holding relevant meeting, Opinions of all experts are synthesized for scientific research elevation. Experts are required to have rich experience and extensive knowledge. At the same time, experts generally have a high degree of education, a keen sense of thinking and judgment, and a strong ability of language expression [7]. The advantage of the expert conference law as follows: Firstly, through drawing on the wisdom of all masses, opinions of experts are expressed and employed sufficiently, which can improve the accuracy of elevation indexes. Secondly, the differences of experts' opinions are showed, which contribute to learn strong points and avoid the shortcomings. Thirdly, having a flexible and convenient application, time and cost can be saved. The disadvantages: Firstly, influenced by opinions of the authorities, the opinions of participants could be changed, which brings inaccurate evaluation outcomes. Secondly, because of personal feelings, some of experts are unwilling to publish opinions differ with others. Thirdly, they are unwilling to revise their opinions that are incomplete or even incorrect because of self-respect [5].

\section{Expert Opinion Method}

In Expert Opinion method (Defir Law), based on systematic procedures, the evaluation team members express opinions using an anonymous method. They have not horizontal contact, only allowed to communicate with investigators. Through filling in the questionnaires and counting people's common understanding and collecting outside advice constantly, A good team communication system can be created eventually the problems can be solved in the management process. In Expert Opinion method, the elevation is completed by using the method of experts' "back-to-back" approach. Without any communication among experts, experts do not know each other. Frequent problems are overcome by this "back-to-back" approach [4]. For example, expert opinions are influenced by the opinions of authorities. The opinions of experts are expressed in true and full. 


\section{E. Social Investigation Law}

In social survey method, reality materials of history and society related to the subjects are collected purposefully in a planning and systematical way, which is often used in research studies. First of all, through the combination of historical research methods, observational research methods, conversations, questionnaires, single case studies, experiments, as well as other methods and ways. There is a planned detailed and systematic understanding for related phenomena. Then, collection data is analyzed, compared, summarized, and synthesized. On this basis, existing contradictions can be discovered and laws in society can be studied [10]. Take the questionnaire survey as an example. The advantages as follows: Firstly, breaking the limitations of time and space, a large number of respondents are investigated, which is convenient to conduct quantitative research on the survey results. Secondly, because of Anonymity, it is more convenient for both investigators and responders, which can eliminates all kinds of interference that may occur in human communication. Thirdly, time, money and human resources are saved. As far as its shortcomings: Firstly, the most remarkable point is that it can only obtain written information in society, but not vivid and concrete situations. Secondly, Due to lacking of flexibility, it is difficult to conduct in-depth evaluation with qualitative methods. Thirdly, based on unified design, it is difficult to adapt to the complex and various evaluation indexes, which brings difficulties to in-depth discussion and qualitative research [6] [7].

\section{THE APPLICATION OF QUANTITATIVE AND \\ QUALITATIVE ANALYSIS METHODS IN THE CONSTRUCTION OF SCIENTIFIC RESEARCH EVALUATION SYSTEM IN COLLEGES AND UNIVERSITIES}

\section{A. Characteristics of Quantitative and Qualitative Analysis Methods}

In qualitative evaluation, the evaluation is based on the description of language. It is mainly based on the intuition and experience of analysts. According to the information of evaluation indexes in past, as well as the latest information, the characteristics and development of indexes are judged [7]. In terms of research methods, inductive methods are often used in qualitative research rather than deductive methods, which emphasizes to observe, describe and understand [8] [10]. On one hand, rich information can be obtained by qualitative analysis; on the other hand, researchers get a large interpretation space, which promotes the creativity of researchers and makes up for the lack of quantitative research.

In Quantitative evaluation, evaluation is based on the description of mathematical linguistic. Establishing statistical data and a mathematical model, indexes and corresponding values are calculated by mathematical models. Due to emphasizing the verification, mathematical analysis and mathematical calculation are applied to each analysis process. On one hand, the results of quantitative analysis are clear and verified; on the other hand, analysis results have no relation with subjectively people's will, which cannot be subjectively affected [6].

\section{B. The Disadvantages of Quantitative and Qualitative Analysis Methods}

At present, there are many advantages for the application of the quantitative evaluation method in the universities research evaluation. For example, improve the efficiency of evaluation, and avoid the interference of some human factors in the evaluation process, etc. However, in most of the current quantitative evaluations in China, it seems to only pay attention to the amount of scientific research rather than quality [8], which results in bad influence and brings "bubble academic". Meanwhile, due to the subjectivity of qualitative evaluation, the unscientific results of scientific research evaluation are uncertain and random. Because of those, the status of scientific research evaluation in China is not satisfactory [10]. Therefore, it is imperative to evaluate the scientific research of universities by combining qualitative and quantitative methods. For example, adding some quantitative evaluation indexes in the qualitative evaluation, the subjectivity and uncertainty in the qualitative evaluation can be avoided in a furthest way. Furthermore, the absolution of indexes in the quantitative evaluation can also be weakened accordingly.

According to people's subjective experience, values, and of thinking way, Qualitative evaluation takes the evaluation object as a concept and uses the analysis and evaluation to explain the characters of the evaluation objects. Compared with quantitative evaluation, qualitative evaluation is simpler and easier. In this kind of evaluation, However, because of the lack of certain standards and the influence of personal feelings, personal opinions advocating authority, acquaintance site, the evaluation results difficult to be fair and just and it is difficult to break academic monopoly. At the same time, the scope of application of qualitative analysis methods will be limited when the results are not theoretical but applicable, or when researching a new discipline or a multidisciplinary rather than a single [7] [9].

According to evaluation data on people or things, Quantitative evaluation quantitatively analyzes and calculates the objectives of evaluation, thereby determining its own value. At the micro level, there are many limitations in the indicators, such as non-representation, unity, and incomparability, etc. Besides, an objectively falling situation occurs at the micro level of evaluation members or a project. The quantitative evaluation method is mostly applied to research evaluation that the macro level of countries comparison and the mid-level of the homogeneous institutions comparison [4].

\section{Application of Quantitative and Qualitative Analysis Methods}

The combination of qualitative and quantitative analysis can be applied to evaluate a certain thing or phenomenon. First of all, analyzing and decomposing the effects to the objective things by targets of research evaluation, a more comprehensive evaluation index system can be created in 
multiple levels [8]. Then the corresponding indicators are assigned values and weight coefficients. Finally, the final evaluation value is obtained through the comprehensive system model, as well as the ranking of corresponding universities, characteristics and drawbacks of each university.

Based on evaluation data of universities ranking in Jilin Province and statistical data of scientific research results, the scientific research evaluation system employ the quantitative model and qualitative analysis method. First of all, researching relates to the correlation, discriminative power and importance of each index in the scientific research evaluation system of colleges and universities, several indicators with the largest impact factors in the ranking provincial universities were selected; Then discussing several indexes with the largest impact factor, the impact of these indexes on the institutions of the universities, provinces, municipalities and private. Thus their effects of indexes are predicted to future development trends in different types of universities [10].

\section{CONCLUSION}

Based on the data of various scientific research evaluation indexes of universities, the quantitative and qualitative analysis methods are applied separately that is mentioned in this paper. Works need to be finished in the next step as follows.

- Apply the quantitative analysis model method to the research of university research evaluation system;

- With the combination of quantitative and qualitative analysis methods, study the role of scientific research evaluation system in the comprehensive development of colleges, and universities and predict its development trend in the future.

It is expected to establish a quantitative analysis model for scientific research evaluation system, and clarify the impact of scientific research evaluation indexes on the ranking of colleges and universities. After that, the basic situation will be discussed for university scientific research evaluation in China from the relationship between university evaluation and scientific evaluation, university scientific research classification evaluation and university scientific research evaluation. All of the above, a theoretical foundation will be laid for further research on university scientific research evaluation.

In the Chinese university rankings sponsored by the China Alumni Association Site, talent cultivation, scientific research and comprehensive reputation are juxtaposed as the first-level indexes of evaluation. In this study, Qualitative and Quantitative Analysis methods of scientific Research Evaluation System can be applied to the two indicators of talent cultivation and comprehensive reputation. Effects of the secondary and tertiary evaluation indexes are explored in the comprehensive development of colleges and universities. At the same time, the quantitative thinking of the scientific research evaluation system can be extended to other fields.

\section{REFERENCES}

[1] Wei Haiyan, Guo Ningsheng, Li Shiting. Analysis of the quality of scientific research managers in research universities $[\mathrm{J}]$. Future and Development, 2011(11):74-76.

[2] Implementation regulations of innovation research Group in Beijing Normal University

[3] Wu Pei. Research on the impact of research evaluation system on the research performance of teachers in research universities [D]. Central South University, 2010.

[4] Wang Yafen. Reflections on improving and perfecting the evaluation system of university scientific research $[\mathrm{J}]$. China Higher Education, 2007(z3): 51-53.

[5] Xu Jie. A simple analysis of modern science and technology[J]. Basic Science of China, 2006, 8(5): 43-45.

[6] [AN Ning, DENG Kai-Xi, MO Lei. Discussion on the construction of university scientific research evaluation system - Taking the construction of scientific research performance evaluation system of south china normal university as an example[J]. Journal of South China Normal University(Natural Science), 2008, 2008(3): 131- 138.

[7] Zhao Denghu. Characteristics and Importance of quantitative analysis[J]. Mathematics Research and Research, 2016(17): 131-131.

[8] Wang Yafen, Jia Lina. Comparasion on evaluation methods of scientific research in universities at home and abroad[J]. Evaluation and Management, 2005(01): 21-24.

[9] Zhu Junwen, Liu Niancai. Deviation and governance of quantitative methods and quality guidance in university scientific research evaluation[J]. Educational Research, 2014(8): 52-59.

[10] Lin Changsheng, Lai Riquan, Zeng Jianwu, et al. Comparison and selection of quantitative evaluation methods for scientific research management performance in Universities[J]. Xiamen Science and Technology, 2006(02): 36-39. 